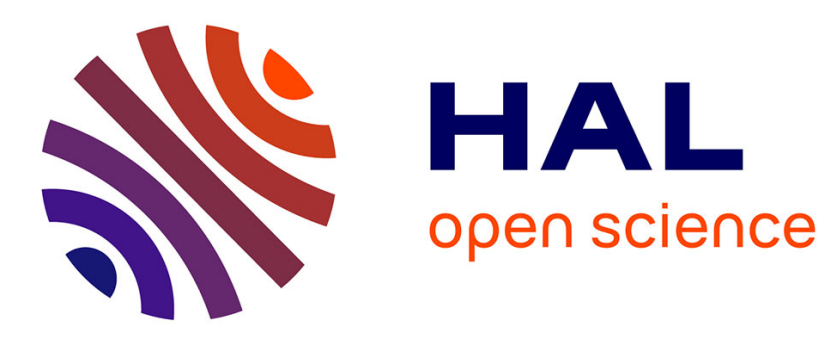

\title{
Parallel Coherent Graph Transformations
}

Thierry Boy de La Tour, Rachid Echahed

\section{To cite this version:}

Thierry Boy de La Tour, Rachid Echahed. Parallel Coherent Graph Transformations. Markus Roggenbach. Recent Trends in Algebraic Development Techniques, 12669, Springer International Publishing, pp.75-97, 2021, Lecture Notes in Computer Science, 978-3-030-73784-9. 10.1007/978-3-030-73785-

6_5. hal-03430231

\section{HAL Id: hal-03430231 \\ https://hal.univ-grenoble-alpes.fr/hal-03430231}

Submitted on 16 Nov 2021

HAL is a multi-disciplinary open access archive for the deposit and dissemination of scientific research documents, whether they are published or not. The documents may come from teaching and research institutions in France or abroad, or from public or private research centers.
L'archive ouverte pluridisciplinaire HAL, est destinée au dépôt et à la diffusion de documents scientifiques de niveau recherche, publiés ou non, émanant des établissements d'enseignement et de recherche français ou étrangers, des laboratoires publics ou privés. 


\title{
Parallel Coherent Graph Transformations
}

\author{
Thierry Boy de la Tour and Rachid Echahed \\ CNRS and University Grenoble Alpes, LIG Lab. Grenoble, France, \\ thierry.boy-de-la-tour@imag.fr \\ rachid.echahed@imag.fr
}

\begin{abstract}
Cellular automata as well as simultaneous assignments in Python can be understood as the parallel application of local rules to a grid or an environment that can be easily represented as an attributed graph. Since the result of such transformations cannot generally be obtained by a sequential application of the involved rules, this situation infringes the standard notion of parallel independence. An algebraic approach with production rules of the form $L \leftarrow K \leftarrow I \rightarrow R$ is adopted and a condition of parallel coherence more general than parallel independence is formulated, that enable the definition of the Parallel Coherent Transformation (PCT). This transformation supports a generalisation of the Parallelism Theorem in the theory of adhesive HLR categories, showing that the PCT yields the expected result of sequential rewriting steps when parallel independence holds. Categories of finitely attributed structures are proposed, in which PCTs are guaranteed to exist. These notions are introduced and illustrated on several detailed examples.
\end{abstract}

\section{Introduction}

Graph transformations 28] constitute a natural extension of string rewriting [3] and term rewriting [2]. Due to the visual and intuitive appearance of their structures, graph rewrite systems play an important role in the modeling of complex systems in various disciplines including computer science, mathematics and biology.

Computing with graphs as first-class citizens requires the use of advanced graph-based computational models. Several approaches to graph transformations have been proposed in the literature, divided in two lines of research: the algebraic approaches (e.g. 28|16) where transformations are defined using notions of category theory, and the algorithmic approaches (e.g. [19]13]) where graph transformations are defined by means of the involved algorithms.

In this context of graph transformations by means of production rules, parallelism is generally understood as the problem of performing in one step what is normally achieved in two or more sequential steps. This is easy when these steps happen to be independent, a situation analogous to the expression $x:=$ $z+1 ; y:=z+2$ that could be executed in any order, hence also in parallel, yielding exactly the same result in each case. If the two steps are not sequentially independent, it may also be possible to synthesize a new production rule 
that accounts for the sequence of transformations in one step (see the Concurrency Theorem in, e.g., [16]). This parallel rule obviously depends on the order in which this sequence in considered, if more than one is possible. As long as parallelism refers to a sequence of transformations, this synthesis can only be commutative if the order of the sequence is irrelevant, i.e., in case of sequential independence.

We can also understand parallelism as a way of expressing a transformation as the simultaneous execution of two (or more) basic transformations. To see how this could be meaningful even when independence does not hold, let us consider a transformation intended to compute the next item in the Fibonacci sequence, given by $u_{n+1}=u_{n-1}+u_{n}$. Since it depends on the two previous items $u_{n-1}$ and $u_{n}$, they need to be saved in two placeholders, say $x$ and $y$ respectively. As the new value $x+y$ of $y$ is computed the old value of $y$ must be simultaneously transfered to $x$. This can be elegantly specified as a multiple assignment $x, y:=y, x+y$ in Python, which can be understood as the parallel evaluation

$$
x:=y \| y:=x+y
$$

It is clear that executing these expressions in sequence in one or the other order yields two different results, hence they are not independent, and that both results are incorrect w.r.t. the intended meaning. This notion of parallelism ought to be commutative in the sense that (1) is equivalent to $y:=x+y \| x:=y$, hence it cannot refer to a sequence of transformations.

Of course (1) can be computed by a sequence of expressions using an extra placeholder (though this breaks the symmetry between the two expressions), or as a single graph transformation rule (see Section 6). The point of the present paper is to define the simultaneous application of possibly non independent graph transformation rules, and to identify the situations in which this is possible.

For the sake of generality a new algebraic approach based on enriched rules is adopted. The motivation of this extension is demonstrated on a detailed example in Section 2. In Section 3, the notion of parallel coherence is introduced, that allows the construction of parallel coherent transformations (PCTs). Section 4 is devoted to the study of PCTs in the theory of adhesive HLR categories. In Section 5, a family of categories is defined in which PCTs are guaranteed to exist. In Section 6, all these notions are illustrated on detailed examples, including a cellular automaton. Related work and concluding remarks are given in Section 7 . The missing proofs can be found in [56].

\section{Weak Spans}

In order to represent the expressions given in (1) as graph transformation rules, the state of the system must be represented as some form of graph. Since we need to hold (and compute with) natural numbers, this obviously requires the use of attributes. A precise definition of attributed graphs is postponed to Section 6 . Example2. Placeholders $x$ and $y$ may be represented as nodes with an arrow from $x$ to $y$, so that placeholder $x$ is identified as the source and $y$ as the sink, hence 
no confusion is possible between the two. The contents of the placeholders are represented as attributes of the corresponding nodes, e.g., $1 \rightarrow 2$ represents the state $(x, y)=(1,2)$. This state is correct in the sense that $(x, y)=\left(u_{n-1}, u_{n}\right)$ for some $n$.

The left-hand side of a production rule corresponding to $y:=x+y$ should then be the graph $L=u \rightarrow v$, where $u$ and $v$ are the contents of placeholders $x$ and $y$ respectively. The right-hand side should ideally be restricted to $R=u+v$, to be matched to placeholder $y$, since $y:=x+y$ has no effect on $x$; the only effect is on $y$ 's content, that should be replaced by $u+v$. In the Double-Pushout approach [11]18, a rule is expressed as a span $L \stackrel{l}{\leftarrow} K \stackrel{r}{\rightarrow} R$, where $l$ specifies what should be removed and $r$ what should be added. Obviously, the content of $y$, and nothing else, has to be removed. This means that $K=u \rightarrow O$.

But then there is no morphism from $K$ to $R$, hence if a span rule is used to express $y:=x+y$ then its right-hand side has to be $u \rightarrow u+v$. Since there must be a matching from the right-hande side of a production rule to the result of the transformation, this means that the value of $x$ cannot change and therefore $x:=y$ cannot be applied simultaneously.

It is therefore necessary to express the lack of effect on $x$ in a weaker sense than as the lack of change (the preservation) of $x$ 's content. Thus, the morphism $r$ should add the content $u+v$ to $y$, and say nothing of $x$ 's content. This is specified by making $r$ match an intermediate graph $I=\bigcirc$ into $R=u+v$. And to make sure that $I$ and $R$ both match to placeholder $y$, a morphism $i$ from $I$ to $K$ that maps $I$ 's node to $K$ 's sink (that stands for $y$ ) is also needed. This leads to the rule below, where $i$ is specified by a dotted arrow in order to avoid any ambiguity.

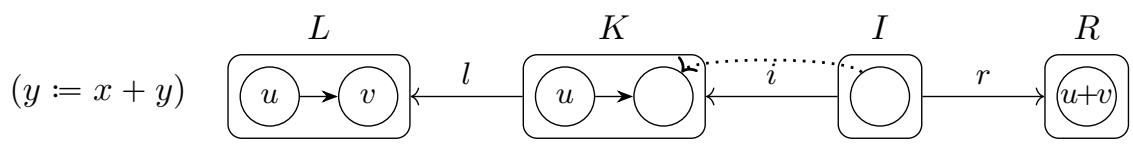

We thus see that the part of $K$ that is not matched by $I$, that can be informally described as $K \backslash i(I)$, is not modified by this rule but can still be modified by another rule, while the part of $K$ that is matched by $I$, i.e., node $y$, is here required to be preserved and therefore cannot be removed by another rule.

Similarly, the rule corresponding to the expression $x:=y$ should be

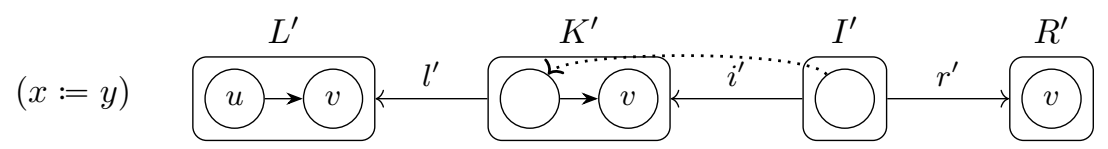

where $i^{\prime}$ maps the node of $I^{\prime}$ to the source node of $K^{\prime}$, that stands for $x$.

A general definition can now be ventured, assuming a suitable category $\mathcal{C}$. 
Definition 1. A weak span is a diagram $L \stackrel{l}{\leftarrow} K \stackrel{i}{\leftarrow} I \stackrel{r}{\rightarrow} R$ in $\mathcal{C}$. Given an object $G$ of $\mathcal{C}$ and a weak span $\rho$, a direct transformation $\gamma$ of $G$ by $\rho$ is a commuting diagram

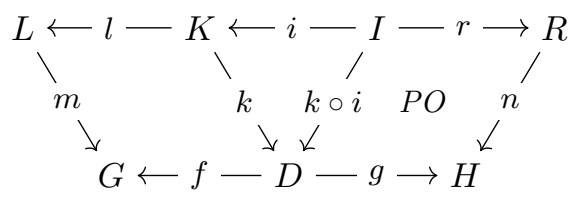

such that $(g, n, H)$ is a pushout of $(I, r, k \circ i)$; we then write $G \stackrel{\gamma}{\Longrightarrow} H$. The object $H$ is called the result and $D$ the context of $\gamma$. Let $\Delta(G, \rho)$ be the set of all direct transformations of $G$ by $\rho$. For a set $\mathcal{R}$ of weak spans, let $\Delta(G, \mathcal{R}) \stackrel{\text { def }}{=}$ $\biguplus_{\rho \in \mathcal{R}} \Delta(G, \rho)$.

If $(f, m, G)$ is a pushout of $(K, l, k)$, then $\gamma$ is called Weak Double-Pushout. Let $\Delta^{\mathrm{PO}}(G, \mathcal{R})$ be the set of Weak Double-Pushouts in $\Delta(G, \mathcal{R})$.

As $\rho$ is part of any diagram $\gamma \in \Delta(G, \rho)$, it is obvious that $\Delta(G, \rho) \cap$ $\Delta\left(G, \rho^{\prime}\right)=\varnothing$ whenever $\rho \neq \rho^{\prime}$. A span can be seen as a weak span where $I=K$ and $i=\mathrm{id}_{K}$, and then a Weak Double-Pushout is a standard Double-Pushout.

Not all elements of $\Delta(G, \rho)$ are usually considered as valid transformations. Many approaches exist to compute the context $D$ from $\rho, G$ and $m$, e.g. [18/10]8]. Each approach corresponds to a particular subset of $\Delta(G, \rho)$ that confers a particular semantics to the production rule $\rho$. Since the parallel transformation defined in Section 3 applies uniformly to all these approaches, it is simply assumed that any element of $\Delta(G, \rho)$ may participate in a parallel transformation.

In the rest of the paper, when we refer to some weak span $\rho$, possibly indexed by a natural number, we will also assume the objects and morphisms $L, K, I$, $R, l, i$ and $r$, indexed by the same number, as given in the definition of weak spans. The same schema will be used for direct transformations and indeed for all diagrams given in future definitions.

\section{Parallel Coherent Transformations}

If we assume direct transformations $\gamma_{1}$ of $G=1 \rightarrow 2$ by $(x:=y)$ and $\gamma_{2}$ of $G$ by $(y:=x+y)$ as in Figure 1, we may then refer to the objects and morphisms involved as $I_{1}, I_{2}, D_{1}, D_{2}, i_{1}, i_{2}$, etc. As stated above, the node that is matched by $I_{2}$, i.e., node $y$, cannot be removed by another rule, hence must belong to $D_{1}$. A parallel transformation is not possible without this condition. This means that there must be a morphism $j_{2}^{1}: I_{2} \rightarrow D_{1}$ that maps $I_{2}$ 's node to the sink in $D_{1}$. Symmetrically, node $x$ matched by $I_{1}$ must belong to $D_{2}$ and there must be a morphism $j_{1}^{2}: I_{1} \rightarrow D_{2}$ that maps $I_{1}$ 's node to the source in $D_{2}$.

Definition 2. Given an object $G$ of $\mathcal{C}$, two weak spans $\rho_{1}$ and $\rho_{2}$, and direct transformations $\gamma_{1} \in \Delta\left(G, \rho_{1}\right)$ and $\gamma_{2} \in \Delta\left(G, \rho_{2}\right)$, we say that $\gamma_{1}$ and $\gamma_{2}$ are parallel coherent if there exist two morphisms $j_{1}^{2}: I_{1} \rightarrow D_{2}$ and $j_{2}^{1}: I_{2} \rightarrow D_{1}$ 


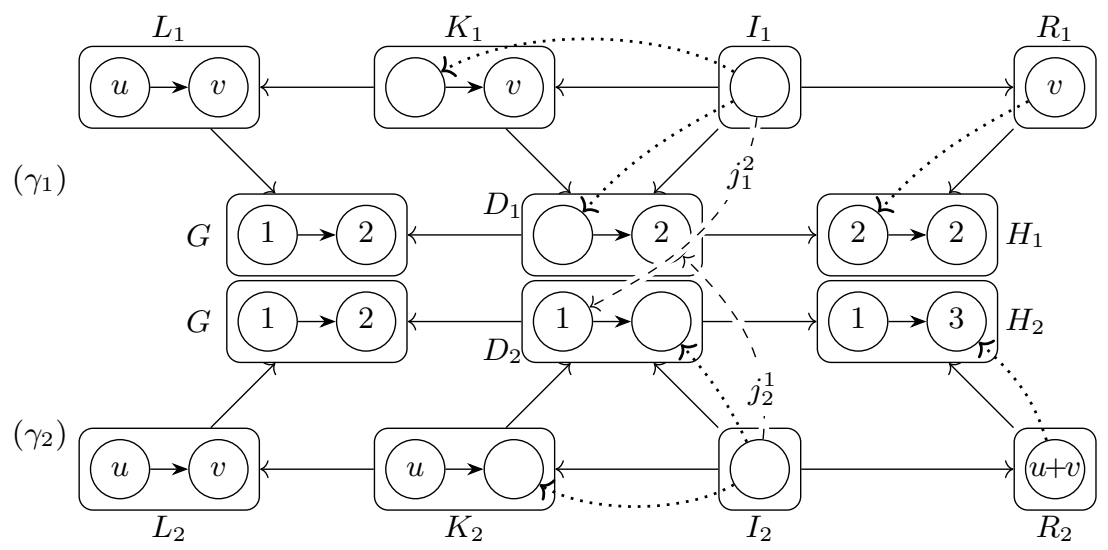

Fig. 1. The direct transformations $\gamma_{1}$ and $\gamma_{2}$

such that the following diagram commutes, i.e., $f_{2} \circ j_{1}^{2}=f_{1} \circ k_{1} \circ i_{1}$ and $f_{1} \circ j_{2}^{1}=$ $f_{2} \circ k_{2} \circ i_{2}$.

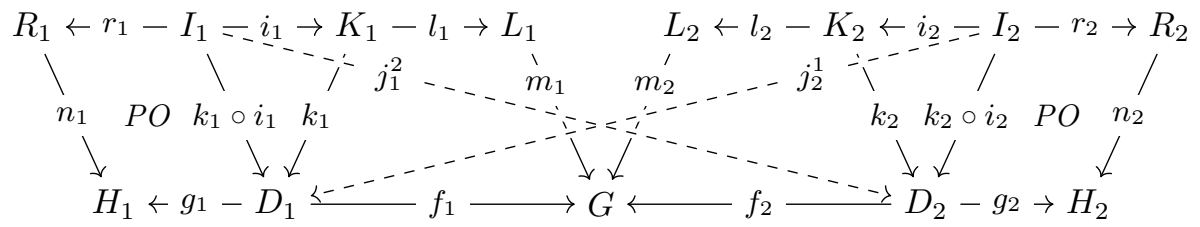

$A$ parallel coherent diagram for $G$ is a commuting diagram $\Gamma$ in $\mathcal{C}$ constituted of diagrams $\gamma_{1}, \ldots, \gamma_{p} \in \Delta(G, \mathcal{R})$ for some $p \geqslant 1$, and morphisms $j_{a}^{b}: I_{a} \rightarrow D_{b}$ for all $1 \leqslant a, b \leqslant p$.

Since $\gamma_{1}, \ldots, \gamma_{p}$ are commuting diagrams, the commuting property of $\Gamma$ amounts to $f_{b} \circ j_{a}^{b}=f_{a} \circ k_{a} \circ i_{a}$ for all $1 \leqslant a, b \leqslant p$. Note that for any $\gamma \in \Delta(G, \mathcal{R})$, the diagram $\Gamma$ constituted of $\gamma$ and morphism $j=k \circ i$ is parallel coherent. For any parallel coherent diagram $\Gamma$, it is obvious that $\gamma_{a}$ and $\gamma_{b}$ are parallel coherent for all $1 \leqslant a, b \leqslant p$, and that

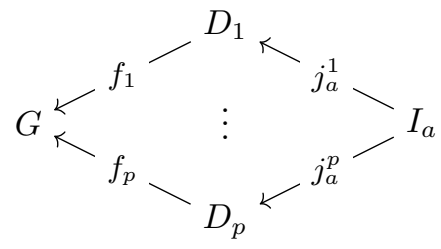

is a sub-diagram of $\Gamma$ for all $1 \leqslant a \leqslant p$, hence commutes.

The transformation of an object by a parallel coherent diagram $\Gamma$ may now be endeavoured. In order to preserve the semantics of the rules, as defined by 


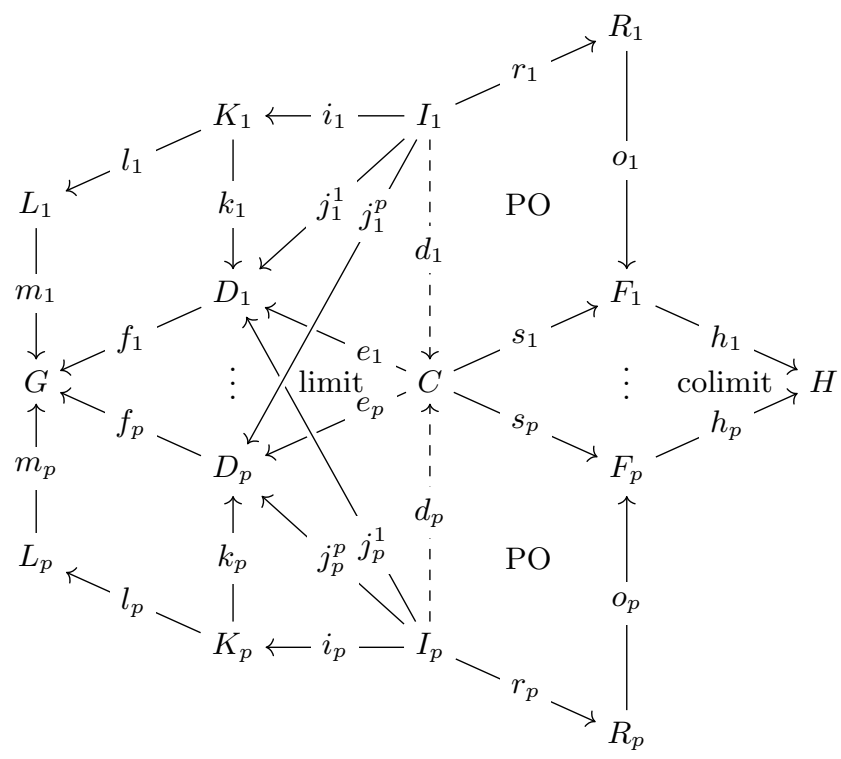

Fig. 2. A parallel coherent transformation (or PCT)

the direct transformations in $\Gamma$, anything that is removed by some direct transformation should be removed from the input $G$, and everything that is added to $G$ by some direct transformation should still be added to $G$.

Definition 3. For any object $G$ of $\mathcal{C}$ and $\Gamma$ a parallel coherent diagram for $G, a$ parallel coherent transformation (or PCT) of $G$ by $\Gamma$ is a diagram as in Figure 2 where:

- $\left(C, e_{1}, \ldots, e_{p}\right)$ is a limit of $\left(f_{1}, \ldots, f_{p}, G\right) ; C$ is called the common context of the PCT,

- for all $1 \leqslant c \leqslant p, d_{c}: I_{c} \rightarrow C$ is the unique morphism such that for all $1 \leqslant a \leqslant p, j_{c}^{a}=e_{a} \circ d_{c}$,

- for all $1 \leqslant a \leqslant p,\left(s_{a}, o_{a}, F_{a}\right)$ is a pushout of $\left(I_{a}, r_{a}, d_{a}\right)$,

- $\left(h_{1}, \ldots, h_{p}, H\right)$ is a colimit of $\left(C, s_{1}, \ldots, s_{p}\right) ; H$ is called the result of the PCT.

If such a diagram exists we write $G \stackrel{\Gamma}{\Longrightarrow} H$.

It is easy to see that $H$ can actually be defined as the colimit of the diagram $\left(C \stackrel{d_{a}}{\longleftarrow} I_{a} \stackrel{r_{a}}{\longrightarrow} R_{a}\right)_{a=1}^{p}$, hence the objects $F_{a}$ could be dispensed with.

If $p=1$ then $C$ is isomorphic to $D_{1}$ and therefore $F_{1}$ and $H$ are isomorphic to $H_{1}$ (the result of the direct transformation $\gamma_{1}$ ). This means that the PCT is a conservative extension of direct transformations, which is obviously a necessary property of parallelism. 
When $p>1$, the left part of the direct transformations $\gamma_{a}$ are preserved, but generally not their results $H_{a}$. This is not surprising since the results of the direct transformations cannot generally be preserved in a parallel transformation (unless they all yield the same result, a rather dull restriction). However, the result $H$ of a PCT does contain images of the right-hand sides $R_{a}$, and more precisely of pushouts along morphisms $r_{a}$, as is the case in direct transformations. Hence the semantics of the individual rules is preserved as much as possible in PCTs: this is why these rules can be said to be applied in parallel. This also means that in practice it is not necessary to compute the pushouts $H_{a}$.

Example 1. Let us consider the following span in the category of graphs

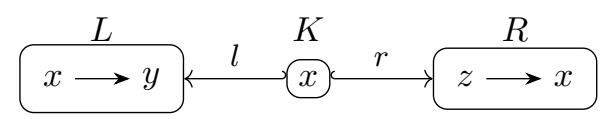

where the hooked arrows are canonical injections, $x$ and $y$ are vertices and the graph arrows are not named since their image by graph morphisms will be uniquely determined by the image of their adjacent vertices. As mentioned in Section 2 this span is a weak span $\rho$ with $K=I$ and $i=\mathrm{id}_{K}$. The left-hand side $L$ has exactly two matches in the graph $G=b \longrightarrow a \longleftarrow c$. Consider the following direct transformation $\gamma_{1}$ of $G$ by $\rho$ :

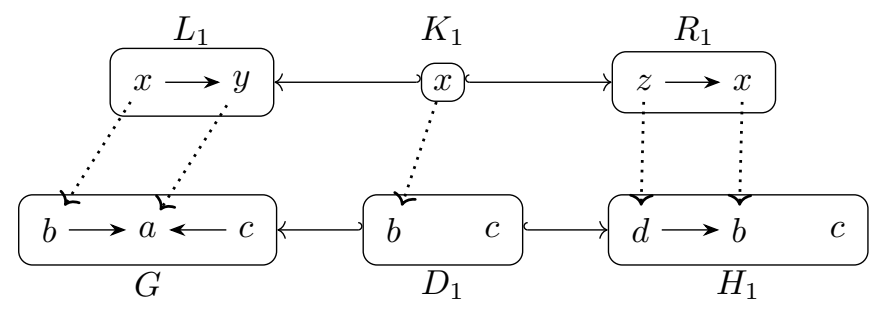

where the dotted arrows indicate the images of vertices by the morphisms that are not canonical injections, i.e., $m_{1}, k_{1}$ and $n_{1}$. In the left part, the vertex $a$ and its two adjacent arrows are removed, in the right part a vertex $d$ and an arrow from $d$ to $b$ are added. This direct transformation is a Sesqui-pushout [10.

Let us next consider the following direct transformation $\gamma_{2}$ of $G$ by $\rho$ :

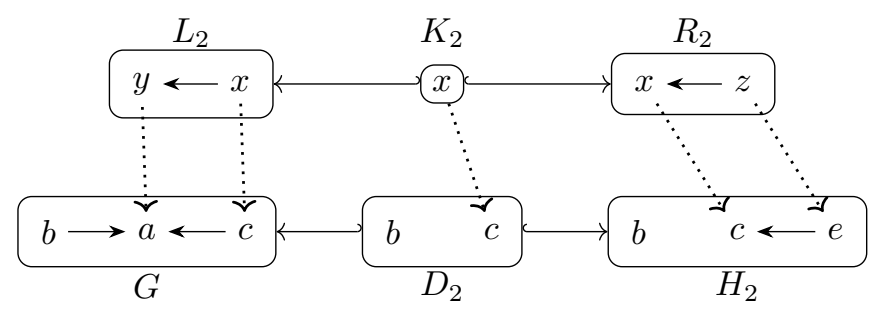

Let $j_{1}^{2}: K_{1} \rightarrow D_{2}$ defined by $j_{1}^{2}(x)=b$, then $f_{2} \circ j_{1}^{2}(x)=b=f_{1} \circ k_{1}(x)$. Similarly, let $j_{2}^{1}: K_{2} \rightarrow D_{1}$ defined by $j_{2}^{1}(x)=c$, then $f_{1} \circ j_{2}^{1}(x)=c=f_{2} \circ k_{2}(x)$, hence $\gamma_{1}$ and $\gamma_{2}$ are parallel coherent. The following diagram is a PCT of $G$ by $\gamma_{1}, \gamma_{2}$. 


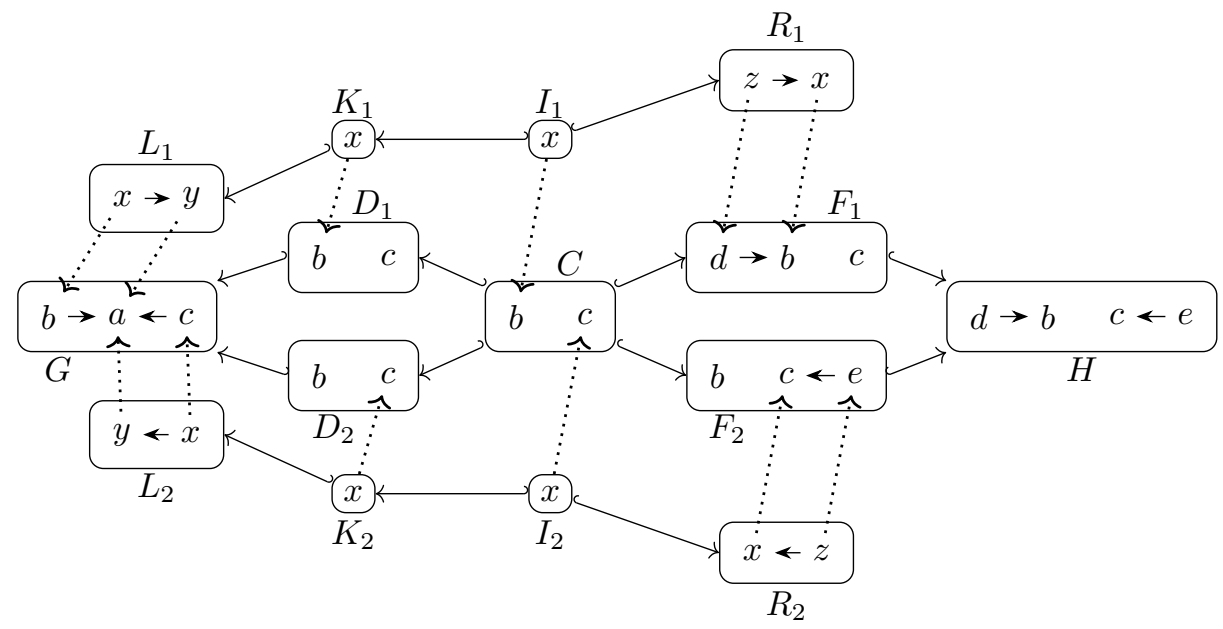

Since $D_{1}=D_{2}=C$ then $F_{1}=H_{1}$ (the result of $\gamma_{1}$ ) and $F_{2}=H_{2}$.

Note that in Definition 3, $\Gamma$ being given, the existence of a PCT diagram depends on the existence of the limit $C$, the pushouts $F_{a}$ and the colimit $H$. Since $H$ may not exist, the existence of a parallel coherent diagram $\Gamma$ is a necessary but by no means a sufficient condition for the existence of a PCT. Two direct derivations may be parallel coherent and still be incompatible, say by writing two different labels on a node that can take at most one. Parallel coherence only involves the left-hand parts of the input derivations.

\section{PCTs in the Theory of Adhesive HLR Categories}

In this section we assume a class of monomorphisms $\mathcal{M}$ of $\mathcal{C}$ that confers $(\mathcal{C}, \mathcal{M})$ a structure of adhesive HLR category. The rather long definition of this concept is not given here; it can be found in [16]. In the results below only the following properties of adhesive HLR categories are used. (Others are used in the missing proofs that can be found in [5].)

1. $\mathcal{M}$ contains all isomorphisms, is closed under composition and under decomposition, i.e., if $g \circ f \in \mathcal{M}$ and $g \in \mathcal{M}$ then $f \in \mathcal{M}$.

2. There are pushouts and pullbacks along morphisms in $\mathcal{M}$, and $\mathcal{M}$ is closed under pushouts and pullbacks, i.e., if $f \in \mathcal{M}$ then there is a pushout square

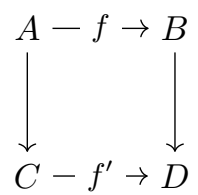

and for all such pushout squares we have $f^{\prime} \in \mathcal{M}$; similarly if $f^{\prime} \in \mathcal{M}$ then there is a pullback square and $f \in \mathcal{M}$ for all such squares. 
In this theory, the morphisms in production rules are elements of $\mathcal{M}$, and the direct derivations are Double Pushouts.

Definition 4. An $\mathcal{M}$-weak span $\rho$ is a weak span whose morphisms l, $i, r$ belong to $\mathcal{M}$. Similarly, an $\mathcal{M}$-span is a span where $l, r \in \mathcal{M}$. The associated span $\check{\rho}$ of $\rho$ is the diagram $L \stackrel{l}{\leftarrow} K \stackrel{r^{\prime}}{\longrightarrow} R^{\prime}$ where $\left(i^{\prime}, r^{\prime}, R^{\prime}\right)$ is a pushout of $(I, i, r)$.

The associated span always exists and is an $\mathcal{M}$-span by the closure properties of $\mathcal{M}$. This association is reflected in the following equivalence of direct derivations.

Lemma 1. For all objects $G, H$ of $\mathcal{C}$ and $\mathcal{M}$-weak span $\rho$, we have

$$
\exists \gamma \in \Delta^{\mathrm{PO}}(G, \rho) \text { s.t. } G \stackrel{\gamma}{\Longrightarrow} H \quad \text { iff } \quad \exists \delta \in \Delta^{\mathrm{PO}}(G, \check{\rho}) \text { s.t. } G \stackrel{\delta}{\Longrightarrow} H .
$$

Proof. Only if part. Since $r \in \mathcal{M}$ there exists a pushout $(g, n, H)$ of $(I, r, k \circ i)$, then $n \circ r=g \circ k \circ i$, hence there is a unique morphism $n^{\prime}: R^{\prime} \rightarrow H$ such that $n^{\prime} \circ i^{\prime}=n$ and $n^{\prime} \circ r^{\prime}=g \circ k$. By pushout decomposition $\left(g, n^{\prime}, H\right)$ is a pushout of $\left(K, r^{\prime}, k\right)$.

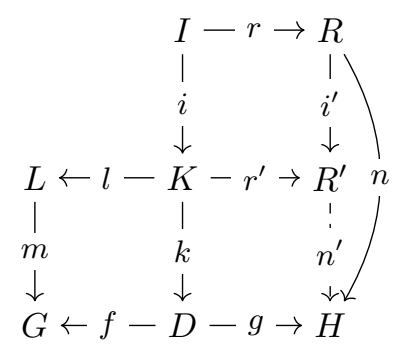

If part. Since $r \in \mathcal{M}$ then $r^{\prime} \in \mathcal{M}$ and hence there exists a pushout $\left(g, n^{\prime}, H\right)$ of $\left(K, r^{\prime}, k\right)$, then by pushout composition $\left(g, n^{\prime} \circ i^{\prime}, H\right)$ is a pushout of $(I, r, k \circ i)$.

This lemma suggests that weak spans can be analyzed with respect to the properties of their associated spans, on which a wealth of results is known.

Definition 5. For any $\mathcal{M}$-weak span $\rho$, object $G$ and $\gamma \in \Delta^{\mathrm{PO}}(G, \rho)$, let $\check{\gamma} \in$ $\Delta^{\mathrm{PO}}(G, \check{\rho})$ be the diagram built from $\gamma$ in the proof of Lemma 1 .

Given $\mathcal{M}$-weak spans $\rho_{1}$ and $\rho_{2}$, an object $G$ of $\mathcal{C}$ and direct transformations $\gamma_{1} \in \Delta^{\mathrm{PO}}\left(G, \rho_{1}\right)$ and $\gamma_{2} \in \Delta^{\mathrm{PO}}\left(G, \rho_{2}\right), \gamma_{1}$ and $\gamma_{2}$ are parallel independent if $\check{\gamma}_{1}$ and $\check{\gamma_{2}}$ are parallel independent, i.e., if there exist morphisms $j_{1}: L_{1} \rightarrow D_{2}$ and $j_{2}: L_{2} \rightarrow D_{1}$ such that $f_{2} \circ j_{1}=m_{1}$ and $f_{1} \circ j_{2}=m_{2}$.

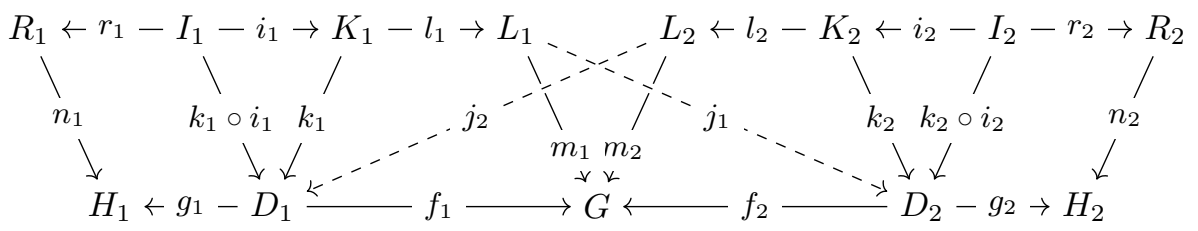


Given direct transformations $\gamma_{1} \in \Delta^{\mathrm{PO}}\left(G, \rho_{1}\right)$ such that $G \stackrel{\gamma_{1}}{\Longrightarrow} H_{1}$ and $\gamma_{2} \in$ $\Delta^{\mathrm{PO}}\left(H_{1}, \rho_{2}\right)$ (with $H_{1} \stackrel{\gamma_{2}}{\Longrightarrow} H_{2}$ ), $\gamma_{1}$ and $\gamma_{2}$ are sequential independent if $\check{\gamma}_{1}$ and $\check{\gamma_{2}}$ are sequential independent, i.e., if there exist morphisms $j_{1}^{\prime}: R_{1}^{\prime} \rightarrow D_{2}$ and $j_{2}^{\prime}: L_{2} \rightarrow D_{1}$ such that $f_{2} \circ j_{1}^{\prime}=n_{1}$ and $g_{1} \circ j_{2}^{\prime}=m_{2}$.

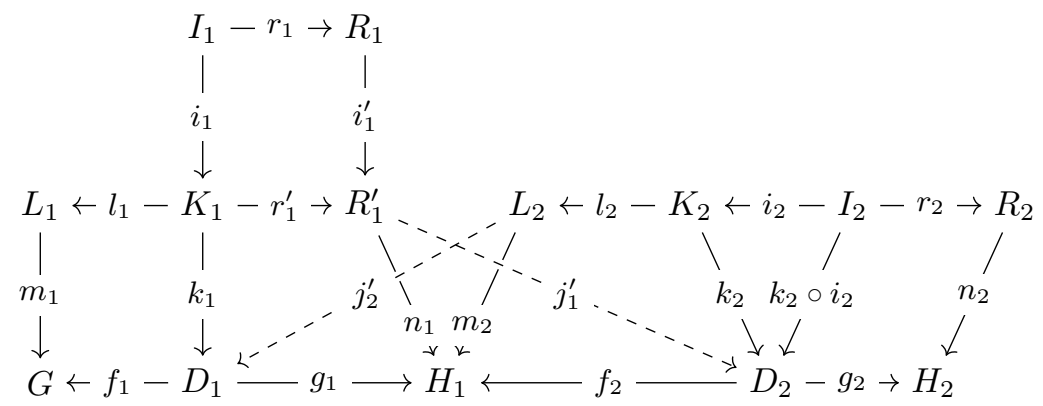

It is obvious that if $\gamma_{1} \in \Delta^{\mathrm{PO}}\left(G, \rho_{1}\right)$ and $\gamma_{2} \in \Delta^{\mathrm{PO}}\left(G, \rho_{2}\right)$ are parallel independent then they are also parallel coherent, and therefore there is a parallel coherent diagram $\Gamma$ corresponding to $\gamma_{1}$ and $\gamma_{2}$ with $j_{1}^{2}=j_{1} \circ l_{1} \circ i_{1}$ and $j_{2}^{1}=j_{2} \circ l_{2} \circ i_{2}$. In the sequel, such diagram $\Gamma$ will be written $\left(\gamma_{1}, \gamma_{2}\right)$.

Theorem 1 (Independent Parallelism Theorem). For any $\mathcal{M}$-weak spans $\rho_{1}, \rho_{2}$, objects $G, H_{1}, H$ and direct transformation $\gamma_{1} \in \Delta^{\mathrm{PO}}\left(G, \rho_{1}\right)$ such that $G \stackrel{\gamma_{1}}{\longrightarrow} H_{1}$, then

1. (analysis) to any $\gamma_{2} \in \Delta^{\mathrm{PO}}\left(G, \rho_{2}\right)$ such that $\gamma_{1}, \gamma_{2}$ are parallel independent and $G \stackrel{\left(\gamma_{1}, \gamma_{2}\right)}{\Longrightarrow} H$, is associated $a \gamma_{2}^{\prime} \in \Delta^{\mathrm{PO}}\left(H_{1}, \rho_{2}\right)$ such that $G \stackrel{\gamma_{1}}{\Longrightarrow}$ $H_{1} \stackrel{\gamma_{2}^{\prime}}{\Longrightarrow} H$ is sequential independent,

2. (synthesis) to any $\gamma_{2}^{\prime} \in \Delta^{\mathrm{PO}}\left(H_{1}, \rho_{2}\right)$ such that $G \stackrel{\gamma_{1}}{\Longrightarrow} H_{1} \stackrel{\gamma_{2}^{\prime}}{\Longrightarrow} H$ is sequential independent is associated a $\gamma_{2} \in \Delta^{\mathrm{PO}}\left(G, \rho_{2}\right)$ such that $\gamma_{1}, \gamma_{2}$ are parallel independent and $G \stackrel{\left(\gamma_{1}, \gamma_{2}\right)}{\Longrightarrow} H$,

3. and these two correspondences are inverse to each other up to isomorphism.

This means that a PCT of $G$ by two parallel independent direct transformations yields a result that can be obtained by a sequence of two direct transformations, in any order (they are sequential independent). This can be interpreted as a result of correctness of PCTs w.r.t. the standard approach to (independent) parallelism of algebraic graph transformations. In this sense, parallel coherence is a conservative extension of parallel independence. Note that Theorem 1 generalizes the standard Parallelism Theorem [16] in two ways: it applies to Weak DoublePushouts and, by using PCTs rather than coproducts, it dispenses with the hypothesis that $\mathcal{C}$ has coproducts compatible with $\mathcal{M}$, i.e., such that $f+g \in \mathcal{M}$ whenever $f, g \in \mathcal{M}$. 
The next result is connected to the notions of derived span of a sequence of direct transformations, that is a shortcut for the sequence (see, e.g., 11, Theorem 3.6.3]). Similarly, it is possible to give a shortcut for a PCT as defined below.

Definition 6. For all objects $G, H$ and weak-spans $\rho$, we write $G \vdash_{\rho} H$ if there exists a diagram $\gamma \in \Delta^{\mathrm{PO}}(G, \rho)$ such that $G \stackrel{\gamma}{\Longrightarrow} H$. We write $G \Vdash_{\mathcal{R}} H$ if there exists a parallel coherent diagram $\Gamma$ with $\gamma_{1}, \ldots, \gamma_{p} \in \Delta^{\mathrm{PO}}(G, \mathcal{R})$ such that $G \stackrel{\Gamma}{\Longrightarrow} H$.

Given a binary relation $\vdash$ between objects of $\mathcal{C}$ and $G, H$ such that $G \vdash H$, we call $\vdash$-derived rule of $G, H$ any span $\sigma$ in $\mathcal{C}$ such that $G \vdash \sigma H$ and for all objects $G^{\prime}, H^{\prime}$ of $\mathcal{C}$, if $G^{\prime} \vdash{ }_{\sigma} H^{\prime}$ then $G^{\prime} \vdash H^{\prime}$.

Note that if $\sigma$ is an $\mathcal{M}$-span then it is also an $\mathcal{M}$-weak span since $\operatorname{id}_{K}$ is an isomorphism and hence belongs to $\mathcal{M}$.

By Lemma 1, for every $\mathcal{M}$-weak span $\rho$ and every objects $G, H$ such that $G \vdash \rho$, the associated span $\check{\rho}$ is a $\vdash{ }_{\rho}$-derived rule of $G, H$. The span $\check{\rho}$ does not depend on $G$ or $H$, but this is not generally true for derived rules.

Theorem 2. For all sets $\mathcal{R}$ of $\mathcal{M}$-weak spans, all objects $G, H$, all parallel coherent diagrams $\Gamma$ and parallel coherent transformations $G \stackrel{\Gamma}{\Longrightarrow} H$, the $\mathcal{M}$ span $G \stackrel{f_{1} \circ e_{1}}{\longleftarrow} \stackrel{h_{1} \circ s_{1}}{\longrightarrow} H$ is $a \Vdash_{\mathcal{R}}$-derived rule of $G, H$.

Hence to any Double-Pushout direct transformation of any object $G^{\prime}$ by this derived rule corresponds a PCT of $G^{\prime}$ that yields the same result.

\section{$5 \quad$ Finitely Attributed Structures}

The problem of the construction of a category suitable for the example of Sections 1 and 2 is now addressed, and more generally the construction of categories where PCTs are guaranteed to exist and can effectively be computed.

This example requires a category of graphs whose nodes can be labelled by zero or one attribute, namely a natural number. More importantly, we saw in Section 2 that morphisms $l$ and $r$ of both rules $(x:=y)$ and $(y:=x+y)$ map an unlabelled node to a labelled node, hence the notion of morphism cannot be strict on labels. This means that the notion of comma category, which is a standard tool for building categories of attributed structures, cannot be used. Another candidate is to use the notion of partially attributed structures, see [12, but the resulting category has few pushouts or colimits. The notion of attributes as special nodes of so-called E-graphs in 16 would better fit our purpose, but PCTs may add an uncontrollable number of edges to an attribute. A more convenient notion of labels as sets of attributes is therefore adopted. This notion encodes in a natural way partial attribution as empty sets and ensures the existence of pushouts and pullbacks (see Lemmas 2 and 3 ).

Another concern is the effective construction of PCTs, hence of finite limits and colimits, which requires to be scrupulous about the finiteness of all structures 
involved. This is particularly important since the attributes belong to infinite sets (e.g. natural numbers), which means that pullbacks of finite attributed graphs may require infinitely many nodes. The proofs of this section can be found in 6.

Definition 7. Let $\mathcal{F}$ be a category with pushouts, pullbacks and a pushoutpreserving functor $V: \mathcal{F} \rightarrow$ FinSets, where FinSets is the category of finite sets. Let $\mathcal{A}$ be a category with a functor $U: \mathcal{A} \rightarrow$ Sets. Let $\mathscr{P}_{<\omega}:$ Sets $\rightarrow$ Sets be the functor which to every set maps the set of its finite subsets. Let $\mathscr{I}:$ FinSets $\rightarrow$ Sets be the canonical injective functor. We write $\mathscr{E} \stackrel{\text { def }}{=} \mathscr{I} \circ V$ and $\mathscr{S} \stackrel{\text { def }}{=} \mathscr{P}_{<\omega} \circ U$.

$A$ finitely attributed structure is a triple $(F, A, f)$ where $F, A$ are objects in $\mathcal{F}, \mathcal{A}$ respectively and $f: \mathscr{E} F \rightarrow \mathscr{S} A$ is a function (a morphism in Sets). A morphism of finitely attributed structures from $(F, A, f)$ to $(G, B, g)$ is a pair $(\sigma, \alpha)$ where $\sigma: F \rightarrow G$ is a morphism in $\mathcal{F}$ and $\alpha: A \rightarrow B$ is a morphism in $\mathcal{A}$ such that $\forall u \in \mathscr{E} F, \mathscr{S} \alpha \circ f(u) \subseteq g \circ \mathscr{E} \sigma(u)$; it is neutral if $A=B$ and $\alpha=\mathrm{id}_{A}$. The identity morphism on $(F, A, f)$ is the morphism $\left(\mathrm{id}_{F}, \mathrm{id}_{A}\right)$. The composition of morphisms $(\sigma, \alpha):(F, A, f) \rightarrow(G, B, g)$ and $(\tau, \beta):(G, B, g) \rightarrow(H, C, h)$ is $(\tau, \beta) \circ(\sigma, \alpha) \stackrel{\text { def }}{=}(\tau \circ \sigma, \beta \circ \alpha)$, that is easily seen to be a morphism from $(F, A, f)$ to $(H, C, h)$. We denote $\mathbf{F i n} \operatorname{Attr}(V, U)$ the category of finitely attributed structures.

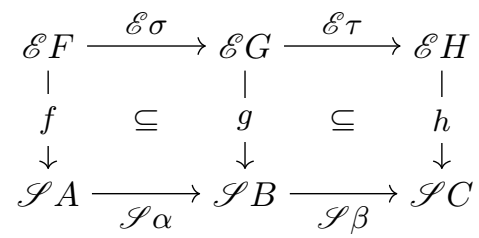

For all $v \in \mathscr{E} G$, we write $\mathscr{E} \sigma^{-1}(v) \stackrel{\text { def }}{=}\{u \in \mathscr{E} F \mid \mathscr{E} \sigma(u)=v\}$.

For instance, $\mathcal{F}$ can be the category of finite graphs and $V$ be the functor that maps any finite graph $G=(V, E, s, t)$ to the direct sum $V+E$ in FinSets, hence $\mathscr{E} G$ is the set of "elements" of $G$. $\mathcal{A}$ can be the category of $\Sigma$-algebras for some signature $\Sigma$, and $U$ the functor that maps any $\Sigma$-algebra $A$ to its carrier set, hence $\mathscr{S} A$ contains the finite subsets of $U A$.

Lemma 2. Let $\left(\sigma, \operatorname{id}_{A}\right):(F, A, f) \rightarrow(G, A, g)$ be a neutral morphism and $(\tau, \alpha):(F, A, f) \rightarrow(H, B, h)$ a morphism with the same domain, let $\left(\sigma^{\prime}, \tau^{\prime}, E\right)$ be a pushout of $(F, \sigma, \tau)$ in $\mathcal{F}$, then $\left(\left(\tau^{\prime}, \alpha\right),\left(\sigma^{\prime}, \operatorname{id}_{B}\right),(E, B, e)\right)$ is a pushout of $\left((F, A, f),\left(\sigma, \operatorname{id}_{A}\right),(\tau, \alpha)\right)$, where for all $x \in \mathscr{E} E$,

$$
e(x)=\left(\bigcup_{v \in \mathscr{E} \tau^{\prime-1}(x)} \mathscr{S} \alpha \circ g(v)\right) \cup\left(\bigcup_{w \in \mathscr{E} \sigma^{\prime-1}(x)} \mathscr{S} \operatorname{id}_{B} \circ h(w)\right) .
$$

Corollary 1. For all integers $p \geqslant 1$, if $s_{a}: C \rightarrow F_{a}$ is a neutral morphism for all $1 \leqslant a \leqslant p$, then there exists a colimit $\left(h_{1}, \ldots, h_{p}, H\right)$ of $\left(C, s_{1}, \ldots, s_{p}\right)$ such that $h_{1}, \ldots, h_{p}$ are neutral morphisms. 
Contrary to pushouts, the construction of pullbacks has to be restricted to the case where both morphisms are neutral.

Lemma 3. Let $\left(\sigma, \mathrm{id}_{A}\right):(G, A, g) \rightarrow(F, A, f)$ and $\left(\tau, \operatorname{id}_{A}\right):(H, A, h) \rightarrow$ $(F, A, f)$ be morphisms and let $\left(E, \sigma^{\prime}, \tau^{\prime}\right)$ be a pullback of $(\sigma, \tau, F)$ in $\mathcal{F}$, then $\left((E, A, e),\left(\sigma^{\prime}, \operatorname{id}_{A}\right),\left(\tau^{\prime}, \mathrm{id}_{A}\right)\right)$ is a pullback of $\left(\left(\sigma, \operatorname{id}_{A}\right),\left(\tau, \operatorname{id}_{A}\right),(F, A, f)\right)$, where for all $x \in \mathscr{E} E, e(x)=g \circ \mathscr{E} \tau^{\prime}(x) \cap h \circ \mathscr{E} \sigma^{\prime}(x)$.

Corollary 2. For all integers $p \geqslant 1$, if $f_{a}: D_{a} \rightarrow G$ is a neutral morphism for all $1 \leqslant a \leqslant p$, then there exists a limit $\left(C, e_{1}, \ldots, e_{p}\right)$ of $\left(f_{1}, \ldots, f_{p}, G\right)$ such that $e_{1}, \ldots, e_{p}$ are neutral morphisms.

With these constructions and their restrictions on morphisms, only transformations of finitely attributed structures that preserve the object $A$ in which the labels are chosen (e.g. the set of natural numbers) can be achieved. This is of course convenient to the example of Section 2, and could be considered sensible in practice.

Definition 8. A weak span $\rho$ in $\boldsymbol{F i n} \operatorname{Attr}(V, U)$ is neutral if its morphisms l, $i$ and $r$ are neutral. For any object $G$, a direct transformation $\gamma \in \Delta(G, \rho)$ is neutral if $\rho$ and its morphisms $f$ and $g$ are neutral. Let $\Delta_{\mathrm{n}}(G, \rho)$ be the set of neutral direct transformations of $G$ by $\rho$.

Note that it is not required of $l, i$ and $r$ to be monomorphisms as in Section 4 .

Theorem 3. For any set $\mathcal{R}$ of neutral weak spans in $\operatorname{Fin} \operatorname{Attr}(V, U)$, for any object $G$ and parallel coherent diagram $\Gamma$ where $\gamma_{1}, \ldots, \gamma_{p} \in \Delta_{\mathrm{n}}(G, \mathcal{R})$, there exists an object $H$, unique up to isomorphism, such that $G \stackrel{\Gamma}{\Longrightarrow} H$.

Proof. Let us prove that a PCT of $G$ by $\Gamma$ can be constructed (see Definition 3). By hypothesis the $f_{a}$ 's are neutral for all $1 \leqslant a \leqslant p$, hence by Corollary 2 there exists a limit $\left(C, e_{1}, \ldots, e_{p}\right)$ of $\left(f_{1}, \ldots, f_{p}, G\right)$, unique up to isomorphism. As $r_{a}$ is neutral, by Lemma 2 there exist pushouts $\left(s_{a}, o_{a}, F_{a}\right)$ of $\left(I_{a}, r_{a}, d_{a}\right)$ where the $s_{a}$ 's are neutral for all $1 \leqslant a \leqslant p$, and they are unique up to isomorphism. By Corollary 1 there exists a colimit $\left(h_{1}, \ldots, h_{p}, H\right)$ of $\left(C, s_{1}, \ldots, s_{p}\right)$, and it is unique up to isomorphism.

A related issue relevant to the Double-Pushout approach is the existence of pushout complements. Provided that a pushout complement exist in $\mathcal{F}$, it is easy to compute at least one pushout complement in $\operatorname{Fin} \operatorname{Attr}(V, U)$, as seen in the following result.

Theorem 4. Let $\left(\sigma, \mathrm{id}_{A}\right):(F, A, f) \rightarrow(G, A, g)$ and $\left(\tau^{\prime}, \alpha\right):(G, A, g) \rightarrow$ $(E, B, e)$ be two morphisms in $\operatorname{FinAttr}(V, U)$, if the left square below is a pushout in $\mathcal{F}$ then so is the right square in $\operatorname{Fin} \operatorname{Attr}(V, U)$, where for all $w \in \mathscr{E} H$

$$
\begin{gathered}
h(w)=\left(e \circ \mathscr{E} \sigma^{\prime}(w) \backslash k(w)\right) \cup \bigcup_{u \in \mathscr{E} \tau^{-1}(w)} \mathscr{S} \alpha \circ f(u) \\
\text { with } k(w) \subseteq \bigcup_{v \in \mathscr{E} \tau^{-1} \circ \mathscr{E} \sigma^{\prime}(w)} \mathscr{S} \alpha \circ g(v) .
\end{gathered}
$$




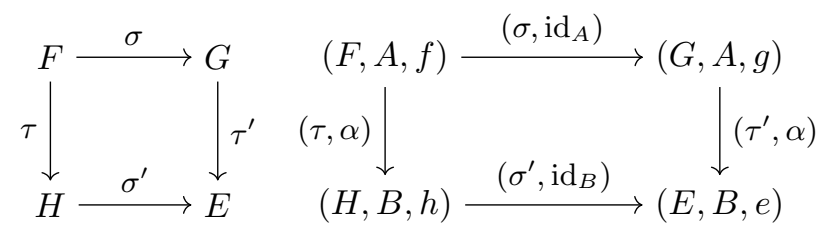

In practice it seems sensible to choose the smallest possible sets for $h(w)$, and hence to take $k(w)=\bigcup_{v \in \mathscr{E} \tau^{\prime-1} \circ \mathscr{E} \sigma^{\prime}(w)} \mathscr{S} \alpha \circ g(v)$. This result also shows that $\operatorname{Fin} \operatorname{Attr}(V, U)$ may not be an adhesive HLR category (where pushout complements are unique), hence that PCTs exist in a larger class of categories than those considered in Section 4

\section{$6 \quad$ Examples}

All the necessary tools are now available to develop in detail the example of Sections 1 and 2 .

Example 2. As suggested above, let $\mathcal{F}$ be the category of finite graphs and $\mathcal{A}$ be the category of $\Sigma$-algebras, where $\Sigma=\{+\}$ and + is a binary function symbol. Among the objects of $\mathcal{A}$ only the standard $\Sigma$-algebra $\mathbb{N}$ and the algebra of $\Sigma$ terms on the set of variables $\{u, v\}$, here denoted $T$, need to be considered. The objects $(F, A, f)$ of $\boldsymbol{F i n A t t r}(V, U)$ will be depicted as graphs indexed by $A$, and since the attributes of nodes will only be $\varnothing$ or singleton ${ }^{1}$ and the attributes of arrows always $\varnothing$, nodes will be represented by circles containing either nothing or an element of $A$ (as in Section 2). The morphisms $(\sigma, \alpha)$ will only be specified as $\alpha$ since the graph morphism $\sigma$ from the domain to the codomain's graphs will either be unique or specified by a dotted arrow (except for the $j$ morphisms). Let $m: T \rightarrow \mathbb{N}$ be the morphism in $\mathcal{A}$ such that $m(u)=1$ and $m(v)=2$.

The finitely attributed graph $G=1 \rightarrow 2$ corresponds to a correct state, and the transformations $\gamma_{1}$ and $\gamma_{2}$ of Figure 1 can be interpreted as diagrams in $\operatorname{Fin} \operatorname{Attr}(V, U)$. They are obviously parallel coherent, hence a PCT of $G$ by $\left\{\gamma_{1}, \gamma_{2}\right\}$ can be constructed, as illustrated in Figure 3 top. The pushouts and pullbacks are computed as in Lemmas 2 and 3 . The result of this transformation is the finitely attributed graph $2 \rightarrow 3 \mathbb{N}$ that corresponds to a correct state.

Observe that these rules (weak spans) have the same left-hand side $L$. The generality of the algebraic approach thus allows us to apply both rules to $L$, which again yields parallel coherent direct transformations and hence the PCT given in Figure 3 bottom (all morphisms are labelled by $\mathrm{id}_{T}$, hence are omitted, as are dotted arrows since they are the same as above). From this PCT the following derived rule can be extracted, which describes the PCT as a single graph transformation rule, already mentioned in Section 1 . Note however that Theorem 2 has been proven in the theory of adhesive HLR categories and not in $\operatorname{FinAttr}(V, U)$.

\footnotetext{
1 This property is not generally true, but happens to be true in this example.
} 


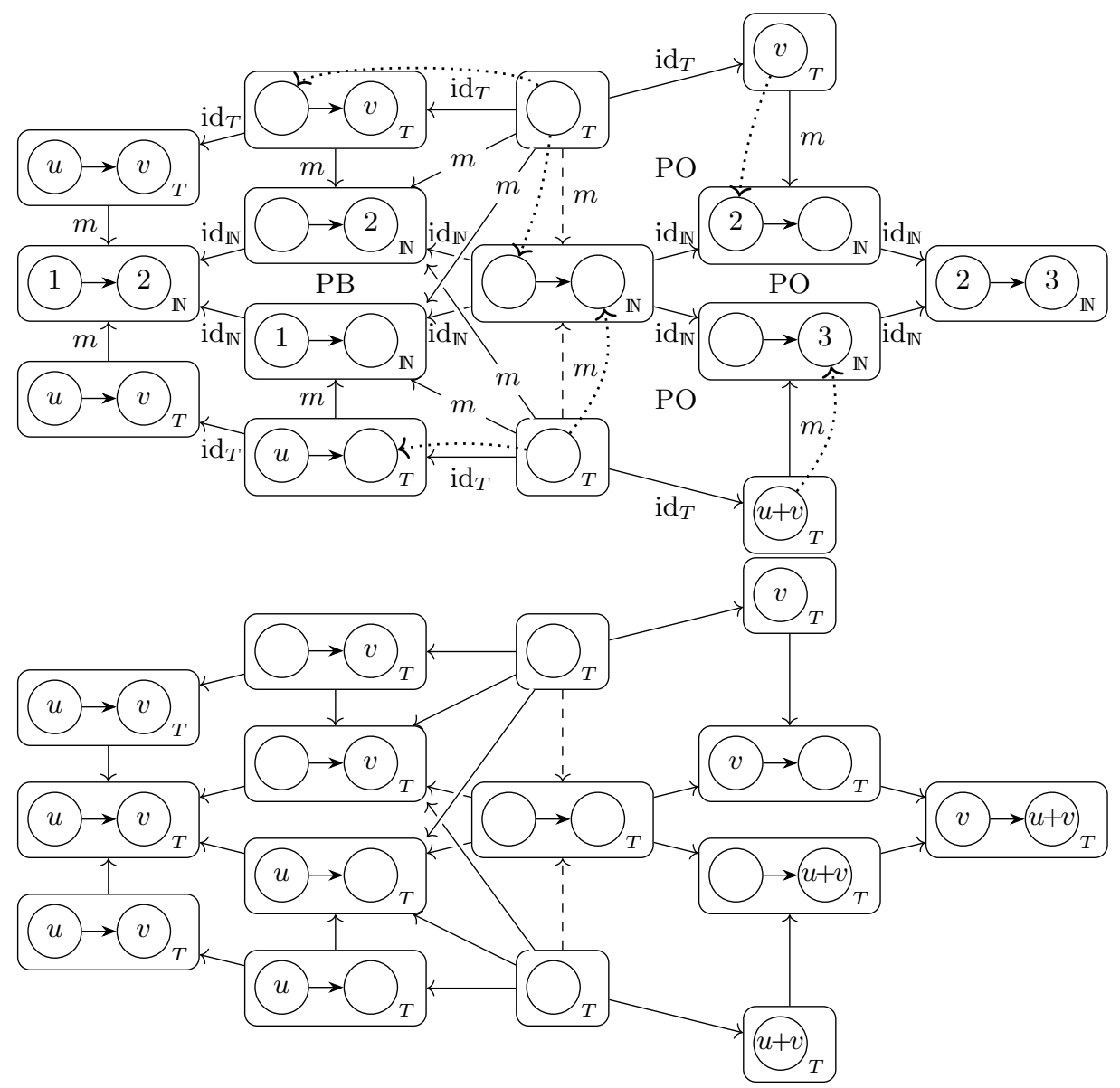

Fig. 3. Two parallel coherent transformations

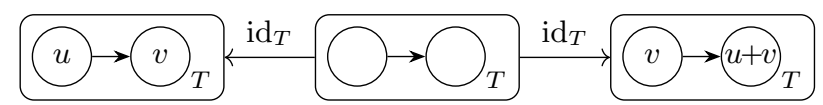

Another important class of examples is provided by cellular automata, where the states of cells at a given generation are computed in parallel from the states of the previous generation. The local transitions may not be independent from each other, as the following example illustrates.

Example 3. The Hex-Ulam-Warburton automaton, see [22, has the same rule as the Ulam-Warburton automaton, namely that a new cell is born if it is adjacent to exactly one live cell, but it grows in the hexagonal grid. The first generations are depicted in Figure 4 and give rise to nice fractal structures as shown in [22].

The six transitions that yield Generation 1 and the 24 that yield Generation 3 are not independent since they cannot be obtained sequentially. In contrast, 


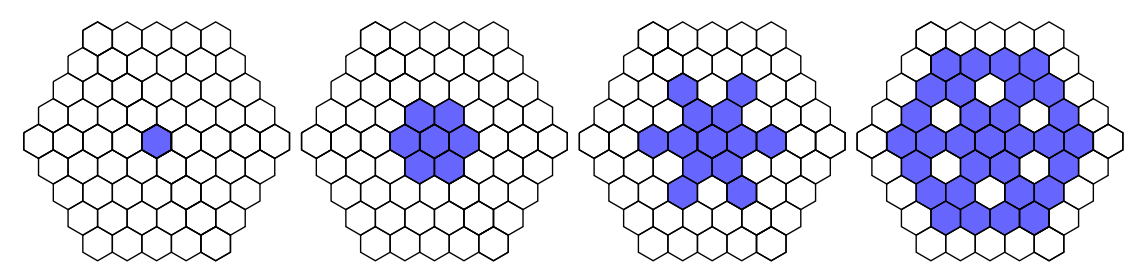

Fig. 4. Generations 0, 1, 2 and 3 of the Hex-Ulam-Warburton automaton

the 6 transitions that yield Generation 2 are independent and can be produced in any order.

In this framework the dead cells are labelled by a singleton, say $\{0\}$ (represented by $\bigcirc$ ), live cells by another singleton, say $\{1\}$ (represented by $\bigcirc$ ), and cells labelled by $\varnothing$ (represented by $\bigcirc$ ) are also needed, hence let $\mathcal{A}$ be the category with one object $\{0,1\}$ and its identity morphism (all morphisms of finitely attributed structures are therefore neutral). Assuming for $\mathcal{F}$ a category of finite hexagonal grids where morphisms map adjacent cells to adjacent cells (or equivalently, a morphism is a translation followed by a rotation of $\frac{k \pi}{3}$ for some $k \in \mathbb{Z}_{6}$ ), the state transitions can be represented by the following weak span

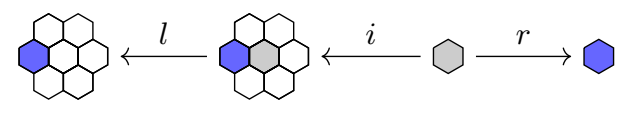

where $i$ maps the cell of $I$ to the center cell of $K$. There are exactly 6 matchings $m_{1}, \ldots, m_{6}$ of $L$ in Generation 0 , centered on the 6 cells adjacent to the live cell and rotated by $\frac{k \pi}{3}$ for $k=0, \ldots, 5$ respectively. Hence there are 6 direct transformations $\gamma_{1}, \ldots, \gamma_{6}$ (not depicted) of Generation 0 that yield the PCT in Figure 5 (where only the matchings $m_{1}$ and $m_{6}$ are depicted).

Note that morphism $j_{1}^{6}$ maps the cell of $I$ to the dead cell (not the empty cell) of $D_{6}$ adjacent to the east border of its live cell, and similarly $j_{6}^{1}$ maps the cell of $I$ to the cell of $D_{1}$ adjacent to the south east border of its live cell, which proves that the pair $\gamma_{1}, \gamma_{6}$ is parallel coherent, and for reasons of symmetry the diagram constituted of $\gamma_{1}, \ldots, \gamma_{6}$ and the morphisms $j_{a}^{b}$ is parallel coherent.

Cellular automata are models of computation that involve infinite cells (located on an infinite grid $\mathbb{Z}^{d}$ ) and each configuration involves infinite transitions. Representing such transformations by means of PCTs implies infinite parallel coherent diagrams. It is of course possible to extend Definition 3 accordingly (see 1, 11.3] for the general definition of a limit). It would then be easy to prove that cellular automata can be represented by PCTs, with a single production rule (see also [4, Theorem 6.3]).

A similar idea has been proposed in 25] by using edge-labelled graphs and rules with positive context where the state of cells are encoded by the label of loops. Rules with positive context seem very close to weak-spans, the only difference is the way direct transformations are defined. We consider here only the DPO approach. 


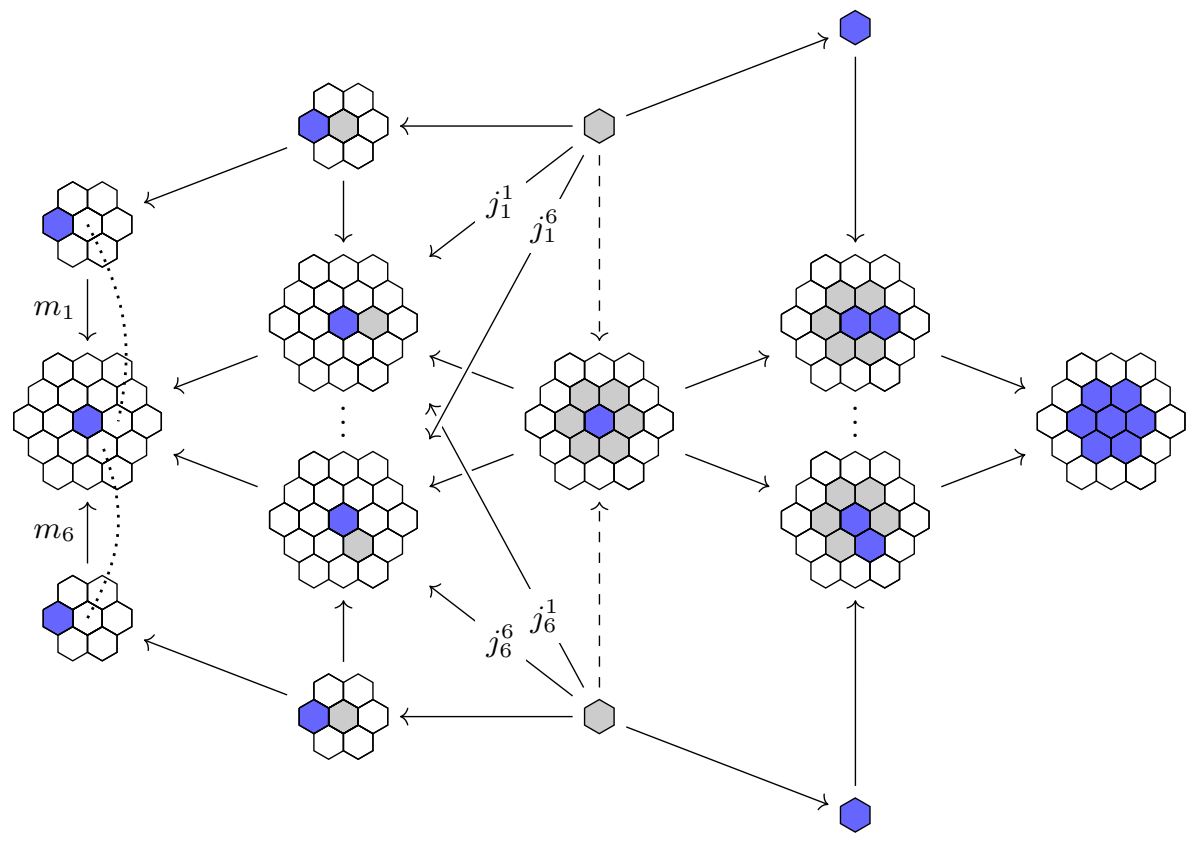

Fig. 5. The parallel coherent transformation of Generation 0

Definition 9. A rule with positive context (or PC-rule) is a diagram

$$
P \stackrel{p}{\longleftarrow} L \stackrel{l}{\longleftarrow} K \stackrel{r}{\rightarrow} R
$$

in $\mathcal{C}$. Given an object $G$ of $\mathcal{C}$ and a $P C$-rule $\pi$, a direct transformation of $G$ by $\pi$ is a commuting diagram

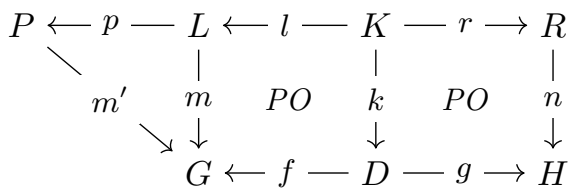

One benefit of PC-rules is that they can be seen as standard span rules together with a very simple form of positive application condition, for which a wealth of results are known, see, e.g., [16, chapter 7]. However, the positive context $P$ may prevent a pushout complement to exist, as we now illustrate.

Example 4. Consider the following weak span

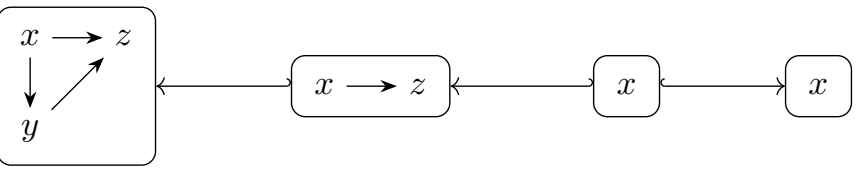


that deletes $y$ (and its two adjacent arrows) and preserves $x$, leaving $z$ to be possibly deleted by another rule. The corresponding PC-rule should be

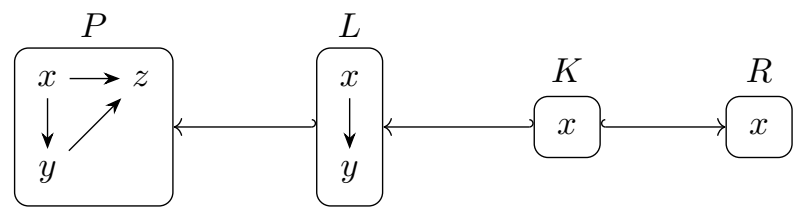

since the deletion of $y$ is enforced by the middle morphism. But this PC-rule can only be applied to a graph $G$ that has an arrow leaving the node matched to $y$, which therefore cannot be deleted according to the gluing condition (see e.g. [16]) by which nodes can only be deleted if all adjacent arrows are explicitly deleted. In fact, this PC-rule cannot by applied to $P$ with the matching id $_{P}$ (for lack of a pushout complement), a problem that never happens with weak-spans, nor indeed with spans.

We therefore see that PCTs generalize cellular automata by allowing not only to modify the state of cells but also their neighborhood frame, and also to delete, merge, create cells, and even to clone cells, as we now illustrate.

Example 5. In the previous examples the morphisms involved in weak spans are always monomorphisms. Non injective morphisms can be used for cloning vertices, and this can also be parallelized as is now illustrated. Let $\gamma_{1}$ be the following direct transformation,

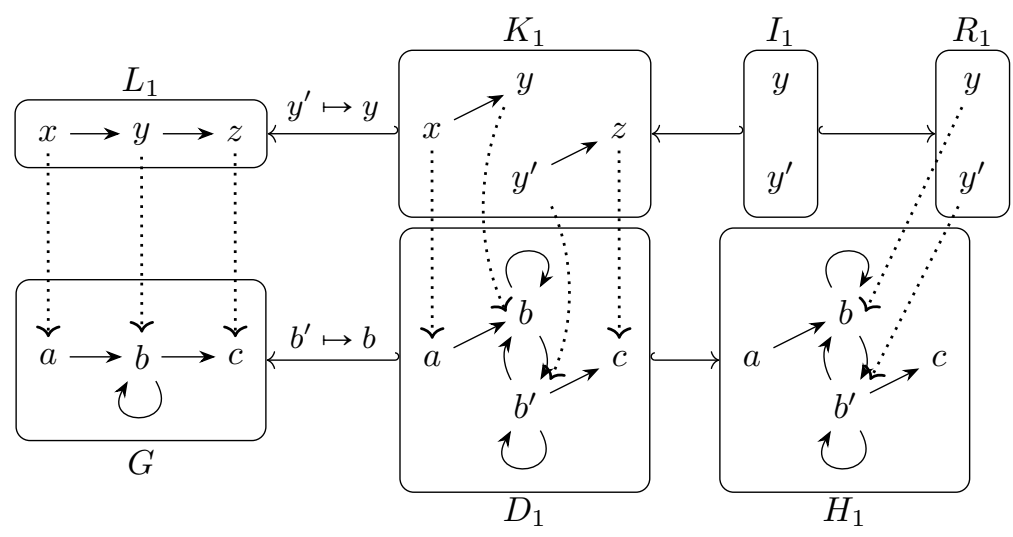

where canonical injections are extended by the indicated function, e.g., $l_{1}(x)=x$, $l_{1}(y)=y, l_{1}(z)=z$ and $l_{1}\left(y^{\prime}\right)=y$. This transformation is a Sesqui-pushout, see [10]: $D_{1}$ is the final pullback complement of $G \stackrel{m_{1}}{\longleftarrow} L_{1} \stackrel{l_{1}}{\longleftarrow} K_{1}$, which means that it is the largest graph such that the left square is a pullback.

Let $\gamma_{2}$ be the following direct transformation of $G$. 


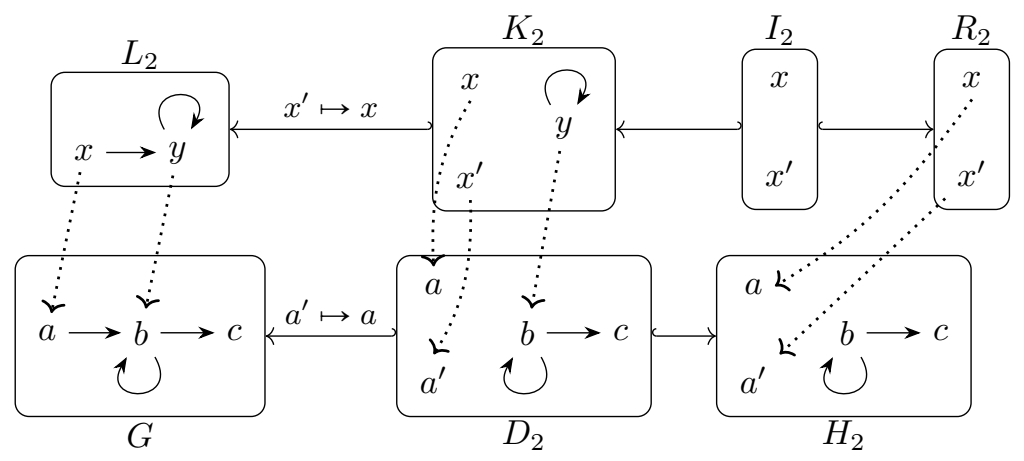

It is easy to see that there is no morphism $j_{1}: L_{1} \rightarrow D_{2}$ such that $f_{2} \circ j_{1}=m_{1}$, hence $\gamma_{1}$ and $\gamma_{2}$ are not parallel independent. But they are parallel coherent: let $j_{1}^{2}: I_{1} \rightarrow D_{2}$ be defined by $j_{1}^{2}(y)=j_{1}^{2}\left(y^{\prime}\right)=b$, and let $j_{2}^{1}: I_{2} \rightarrow D_{1}$ be defined by $j_{2}^{1}(x)=j_{2}^{1}\left(x^{\prime}\right)=a$, we have $f_{2} \circ j_{1}^{2}=f_{1} \circ k_{1} \circ i_{1}$ and $f_{1} \circ j_{2}^{1}=f_{2} \circ k_{2} \circ i_{2}$. A PCT can therefore be built from $\gamma_{1}, \gamma_{2}, j_{1}^{2}, j_{2}^{1}$. Since $I_{1}=R_{1}$ and $r_{1}=\mathrm{id}_{I_{1}}$, then $o_{1}=d_{1}, F_{1}=C$ and $s_{1}=\mathrm{id}_{C}$. Similarly $s_{2}=\mathrm{id}_{C}$ and therefore $H=C$; the result of the PCT is the common context and is easy to compute as the pullback of $\left(f_{1}, f_{2}, G\right)$.

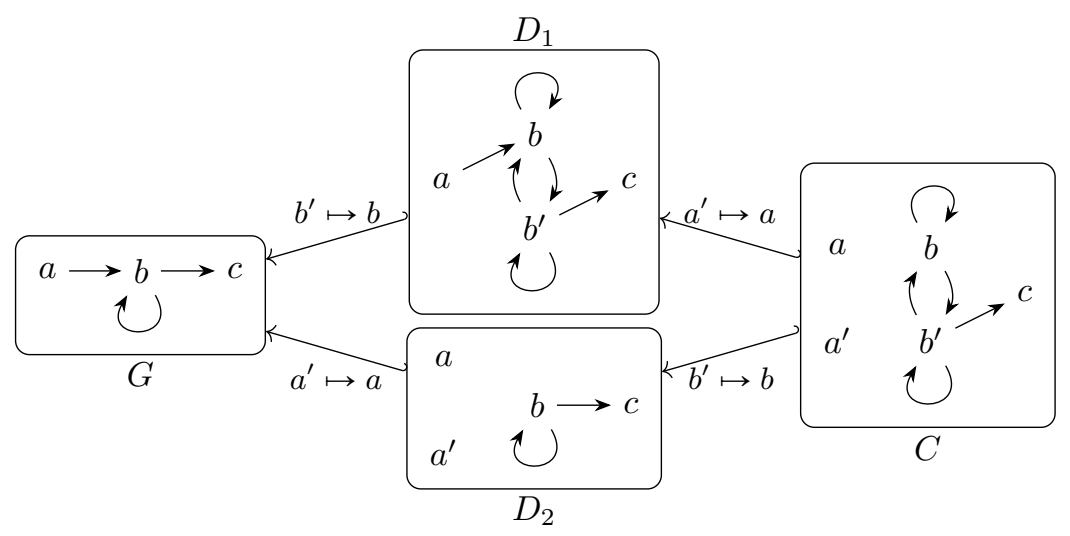

We therefore see that the cloning of $a$ performed by one rule and the cloning of $b$ performed by the other rule can be performed in parallel by means of a PCT.

We also see that, with $j_{2}: L_{2} \rightarrow D_{1}$ defined by $j_{2}(x)=a$ and $j_{2}(y)=b$ we have $f_{1} \circ j_{2}=m_{2}$. This means that the second rule can be applied to the result $H_{1}$ of $\gamma_{1}$; this yields a direct transformation $\gamma_{2}^{\prime}$. The reader may check that the result of $\gamma_{2}^{\prime}$ is exactly the result of the PCT. In this sense, this PCT yields a correct result. 


\section{Conclusion and Related Work}

Parallel graph rewriting has already been considered in the literature. In the mid-seventies, H. Ehrig and H.-J. Kreowski [17 tackled the problem of parallel graph transformations and introduced the notion of parallel independence. This pioneering work has been considered for several algebraic graph transformation approaches, see [15] as well as the more recent contributions [7 26 25]. At almost the same period, parallel graph transformations has been used as an extension of L-systems 29131] as was proposed in, e.g., [21]. This stream of work departs drastically from the present one, where parallel derivations are not meant to be sequentialized.

In [27, chapter 14], parallel graph transformations have been studied in order to improve the operational semantics of the functional programming language CLEAN [20, where parallelism is considered under an interleaving semantics of parallelism. Such is the case of other frameworks [14|25|24] where massive parallel graph transformations are defined in order to simulate sequential rewriting.

Non independent parallelism has been considered in the Double-Pushout approach, see e.g. 30 where rules can be amalgamated by agreeing on common deletions and creations. This approach is formulated on span rules where the notion of parallel coherence loses much of its expressive power. Besides, amalgamation may not preserve the semantics of the rules that are amalgamated, in the sense that an item that should be deleted according to an input rule and a matching may not be deleted by an amalgamated rule.

In [23, a framework based on the algebraic Single-Pushout approach has been proposed where conflicts between parallel transformations are allowed but requires the user to solve them by providing the right control flow.

The present work stems from [4] where an algorithmic framework is proposed for rule-based deterministic parallel transformations of graphs whose vertices and arrows are mapped to sets of attributes. The rules are triples $(L, K, R)$ where $K$ is a subgraph of $L$ and the intersection of $L$ and $R$ (that stands for the object $I$ in a weak span) is a subgraph of $K$. Parallel coherence is replaced with a more general effective deletion property that distinguishes graph items (vertices and arrows) from attributes. It is shown that every cellular automaton can be represented by a single rule and a class of infinite graphs that correspond to configurations of the automaton (where cells are vertices attributed with a state), so that the parallel transformations of these graphs is the transition function of the automaton.

The introduction of the notion of weak span is key in our contribution which gives a new insight in the study of parallel graph transformation. Particularly, the object $D$ in Definition 1 could be constructed following different algebraic methods such as DPO [18] or SqPO [10]. Extension to recent approaches such as AGREE [8] or PBPO [9] is rather straightforward. This opens the possibility to integrate, in one parallel step, rules written in different approaches. Future work include further analysis of PCTs and particularly the development of dedicated verification techniques. 


\section{References}

1. Adámek, J., Herrlich, H., Strecker, G.E.: Abstract and Concrete Categories - The Joy of Cats. Online Edition (2004), http://katmat.math.uni-bremen.de/acc/

2. Baader, F., Nipkow, T.: Term Rewriting and All That. Cambridge University Press (1998)

3. Book, R.V., Otto, F.: String-Rewriting Systems. Texts and Monographs in Computer Science, Springer (1993)

4. Boy de la Tour, T., Echahed, R.: Parallel rewriting of attributed graphs. Theoretical Computer Science (2020). https://doi.org/10.1016/j.tcs.2020.09.025, (to appear)

5. Boy de la Tour, T.: Parallelism theorem and derived rules for parallel coherent transformations. CoRR (abs/1907.06585) (2019)

6. Boy de la Tour, T., Echahed, R.: True parallel graph transformations: an algebraic approach based on weak spans. CoRR (abs/1904.08850) (2019)

7. Corradini, A., Duval, D., Löwe, M., Ribeiro, L., Machado, R., Costa, A., Azzi, G., Bezerra, J.S., Rodrigues, L.M.: On the essence of parallel independence for the double-pushout and sesqui-pushout approaches. In: Heckel, R., Taentzer, G. (eds.) Graph Transformation, Specifications, and Nets - In Memory of Hartmut Ehrig. LNCS, vol. 10800, pp. 1-18. Springer (2018)

8. Corradini, A., Duval, D., Echahed, R., Prost, F., Ribeiro, L.: AGREE - algebraic graph rewriting with controlled embedding. In: 8th ICGT. LNCS, vol. 9151, pp. 35-51. Springer (2015)

9. Corradini, A., Duval, D., Echahed, R., Prost, F., Ribeiro, L.: The pullback-pushout approach to algebraic graph transformation. In: 10th ICGT. LNCS, vol. 10373, pp. 3-19. Springer (2017)

10. Corradini, A., Heindel, T., Hermann, F., König, B.: Sesqui-pushout rewriting. In: ICGT 2006. LNCS, vol. 4178, pp. 30-45. Springer (2006)

11. Corradini, A., Montanari, U., Rossi, F., Ehrig, H., Heckel, R., Löwe, M.: Algebraic approaches to graph transformation - part I: basic concepts and double pushout approach. In: Rozenberg [28], pp. 163-246

12. Duval, D., Echahed, R., Prost, F., Ribeiro, L.: Transformation of attributed structures with cloning. In: Gnesi, S., Rensink, A. (eds.) Fundamental Approaches to Software Engineering - 17th International Conference, FASE 2014. LNCS, vol. 8411, pp. 310-324. Springer (2014)

13. Echahed, R.: Inductively sequential term-graph rewrite systems. In: ICGT 2008. LNCS, vol. 5214, pp. 84-98. Springer (2008)

14. Echahed, R., Janodet, J.: Parallel admissible graph rewriting. In: Recent Trends in Algebraic Development Techniques, 13th International Workshop WADT'98, Selected Papers. LNCS, vol. 1589, pp. 122-137. Springer (1999)

15. Ehrig, H., Kreowski, H.J., Montanari, U., Rozenberg, G. (eds.): Handbook of Graph Grammars and Computing by Graph Transformations, Volume 3: Concurrency, Parallelism and Distribution. World Scientific (1999)

16. Ehrig, H., Ehrig, K., Prange, U., Taentzer, G.: Fundamentals of Algebraic Graph Transformation. Monographs in Theoretical Computer Science. An EATCS Series, Springer (2006)

17. Ehrig, H., Kreowski, H.: Parallelism of manipulations in multidimensional information structures. In: Mathematical Foundations of Computer Science. LNCS, vol. 45, pp. 284-293. Springer (1976) 
18. Ehrig, H., Pfender, M., Schneider, H.J.: Graph-grammars: An algebraic approach. In: 14th Annual Symposium on Switching and Automata Theory, Iowa City, Iowa, USA, October 15-17, 1973. pp. 167-180 (1973). https://doi.org/10.1109/SWAT.1973.11

19. Engelfriet, J., Rozenberg, G.: Node replacement graph grammars. In: Rozenberg 28], pp. 1-94

20. Group, S.T.R.: The Clean Home Page. Radboud University, Nijmegen

21. II, K.C., Lindenmayer, A.: Parallel graph generating and recurrence systems for multicellular development. International Journal of General Systems 3(1), 53-66 (1976). https://doi.org/10.1080/03081077608934737

22. Khovanova, T., Nie, E., Puranik, A.: The Sierpinski triangle and the UlamWarburton automaton. Tech. Rep. arXiv:1408.5937v1, ArXiV (August 2014), https://arxiv.org/format/1408.5937

23. Kniemeyer, O., Barczik, G., Hemmerling, R., Kurth, W.: Relational growth grammars - A parallel graph transformation approach with applications in biology and architecture. In: Third International Symposium AGTIVE, Revised Selected and Invited Papers. pp. 152-167 (2007)

24. Kreowski, H., Kuske, S.: Graph multiset transformation: a new framework for massively parallel computation inspired by DNA computing. Natural Computing 10(2), 961-986 (2011)

25. Kreowski, H., Kuske, S., Lye, A.: A simple notion of parallel graph transformation and its perspectives. In: Graph Transformation, Specifications, and Nets - In Memory of Hartmut Ehrig. LNCS, vol. 10800, pp. 61-82. Springer (2018)

26. Löwe, M.: Characterisation of parallel independence in AGREE-rewriting. In: 11th ICGT. LNCS, vol. 10887, pp. 118-133. Springer (2018)

27. Plasmeijer, R., Eekelen, M.V.: Functional Programming and Parallel Graph Rewriting. Addison-Wesley Longman Publishing Co., Inc., Boston, MA, USA, 1st edn. (1993)

28. Rozenberg, G. (ed.): Handbook of Graph Grammars and Computing by Graph Transformations, Volume 1: Foundations. World Scientific (1997)

29. Rozenberg, G., Salomaa, A.: The book of L. Springer (1986)

30. Taentzer, G.: Parallel high-level replacement systems. Theoretical Computer Science 186, 43-81 (1997)

31. Wolfram, S.: A new kind of science. Wolfram-Media (2002) 